EPJ manuscript No.

(will be inserted by the editor)

\title{
Jet Physics in ALICE
}

\author{
Mercedes López Noriega for the ALICE Collaboration
}

Received: date / Revised version: date

\begin{abstract}
The strong suppression of high- $p_{\mathrm{T}}$ hadrons observed in heavy ion collisions at RHIC indicates the interaction of high energy partons with a dense colored medium prior to hadronization. We review the main results from the high- $p_{\mathrm{T}}$ hadron analysis at RHIC and what they tell us about the medium. We then concentrate on the new possibilities that the wider kinematic range at the LHC will offer and how they will help us to better characterize the medium produced in these collisions.
\end{abstract}

PACS. PACS-key discribing text of that key - PACS-key discribing text of that key

\section{Introduction}

In relativistic heavy ion collisions, large transverse momentum partons result from the initial hard scattering of nucleon constituents. After a hard scattering, the parton fragments to create a high energy collimated spray of particles, that is usually called a jet. These partons will travel through what is predicted to be a dense colored medium, 1 and there they are expected to lose energy via medium 7 induced gluon radiation [1,2, this is usually called "jet quenching". The magnitude of this energy loss is predicted - to depend strongly on the gluon density of the medium. $\because$ Therefore, measurements on how quenching changes the structure of the jet and its fragmentation function will reveal information about the QCD medium created in these - collisions.

We start by giving a brief summary about the indications of partonic energy loss found in the analysis of high$p_{\mathrm{T}}$ hadrons at RHIC. We then discuss the advantages of reconstructing the full jet and how this will be done in ALICE. We finish by describing the different jet structure observables that we will study and what they will tell us about the medium.

\section{High- $p_{\mathrm{T}}$ Analysis at RHIC}

Clear evidences of partonic energy loss in heavy ion collisions have been seen at RHIC. There is a strong suppression of high- $p_{\mathrm{T}}$ hadrons in central AuAu collisions at $\sqrt{s_{\mathrm{NN}}}=200 \mathrm{GeV}$ as can be seen in the nuclear modification factor $R_{\mathrm{AA}}$ for charged hadrons and neutral pions [3, 4,5,6]. $R_{\mathrm{AA}}$ measures the deviation of heavy-ion collisions from a simple incoherent superposition of pp collisions. The fact that this factor is well below unity for $p_{\mathrm{T}}>5 \mathrm{GeV} / c$ indicates that hadron production is suppressed by about 5 in central $\mathrm{AuAu}$ collisions relative to pp collisions at that large $p_{\mathrm{T}}$ [3, 4,5,6]. Such suppression is however not observed in central dAu collisions at the same collision energy indicating that the suppression observed in central AuAu collisions is due to final-state interactions of the high energetic partons with the dense system created in these collisions.

In the distribution of the relative azimuth $\Delta \phi$ between pairs of charged hadrons we can see a clear dijet signal in pp and dAu collisions as two distinct back to back Gaussian peaks [3]. No such signal is observed in central AuAu collisions where there is a strong suppression of the leading fragments of the recoiling jet. Once more, the fact that this suppression is not observed in $\mathrm{dAu}$ collisions indicates that the suppression seen in $\mathrm{AuAu}$ collisions is due to final state interactions of the high-energy partons with the dense colored medium created in AuAu collisions.

Different models have incorporated energy loss via medium induced gluon radiation in perturbative QCD calculations. This has been done in two different ways, as multiple soft interactions (BDMPS) [7 and as few hard scatterings (GLV) 8]. In the BDMPS model the density of the medium is characterizes by the transport coefficient $\hat{q}$. Physically, $\hat{q}$ is the mean squared transverse momentum generated by interactions with the medium per unit of path length $\lambda$. In the high energy limit and for a static medium the average radiative energy loss $\Delta E$ is proportional to $\hat{q}$. For cold nuclear matter $\hat{q} \simeq 0.05 \mathrm{GeV}^{2} / \mathrm{fm}$.

In order to be able to reproduce the results from RHIC, such as the suppression observed in $R_{\mathrm{AA}}$, these models require that the early phase of the collision has a gluon density around 30-50 times that of cold nuclear matter, which in the case of the BDMPS model means a $\hat{q}$ between 5 and $15 \mathrm{GeV}^{2} / \mathrm{fm}$. 


\section{Jet Reconstruction}

As mentioned in the previous section, the effects of the medium on the propagation of high-energy partons is studied by the analysis of the leading particles of the jets through the $p_{\mathrm{T}}$ spectra of hadrons and through the azimuthal distribution of pairs of high- $p_{\mathrm{T}}$ particles. However, the study of the leading particles of the jets has its limitation and they become fragile as a probe. For extreme quenching scenarios, one observes particle emission predominantly from the surface and therefore the sensitivity of the leading particles to the region of highest energy density is very limited. As a consequence of this dominance of the skin-emission, the nuclear modification factor has very little sensitivity to the medium properties: it is almost independent of $p_{\mathrm{T}}$ and its dependence on $\hat{q}$ becomes weaker as $\hat{q}$ increases making the characterization of the medium based on this observable very difficult 9 .

However, the full reconstruction of jets is, in principle, free of such a bias and it allows the measurement of the original parton 4-momentum and the transverse and longitudinal structure of the jet. One can then study the properties of the medium through the modifications on the jet structure. Partonic energy loss will manifest in a decrease on the number of particles carrying a high fraction $z$ of the jet energy, and on the appearance of radiated energy via an increase on the number of particles with low $z$ values. In addition, a broadening of the distribution of jet-particle momenta perpendicular to the jet axis is expected. This broadening is predicted to be directly related to the color density of the medium [10].

\subsection{Jet Reconstruction in ALICE}

The ALICE detector is a general purpose heavy-ion experiment designed to cope with the highest particle multiplicities predicted for $\mathrm{PbPb}$ collisions at the LHC of $d N_{\mathrm{ch}} / d y$ up to 8000 [11. The detector consists of a central part with full azimuthal coverage in the pseudorapidity region $|\eta|<0.9$ to measure hadrons, electrons and photons, and a forward dimuon spectrometer to measure muons. The central region is located inside a solenoidal magnet which allows the identification of high- $p_{\mathrm{T}}$ charged hadrons with a momentum resolution better than $10 \%$ up to $100 \mathrm{GeV}$ [12]. There is a proposed electromagnetic calorimeter that will improve jet energy resolution and will add trigger capabilities. It will have an azimuthal coverage between 0 and $110^{\circ}$ within $|\eta|<0.7[13$.

Figure 1 shows the predicted annual jet yields vs. energy within the fiducial region of ALICE $|\eta|<0.5$ for the $10 \%$ most central and minimum bias $\mathrm{PbPb}$ collisions for a typical luminosity at the LHC $\left(5 \times 10^{26} \mathrm{~cm}^{-2} \mathrm{~s}^{-1}\right)$ for one effective month of running $\left(10^{6} \mathrm{~s}\right)$. For $E_{\mathrm{T}}<100 \mathrm{GeV}$ the jet rate $>1 \mathrm{~Hz}$ is high enough to collect a sample of $O\left(10^{4}\right)$ jets. For $E_{\mathrm{T}}>100 \mathrm{GeV}$ triggering will be necessary to collect jet enriched data.

As will be shown below, jets with $E_{\mathrm{T}}>50 \mathrm{GeV}$ will allow full reconstruction of the hadronic jets, even in the underlying heavy-ion environment. Experimentally, jets in

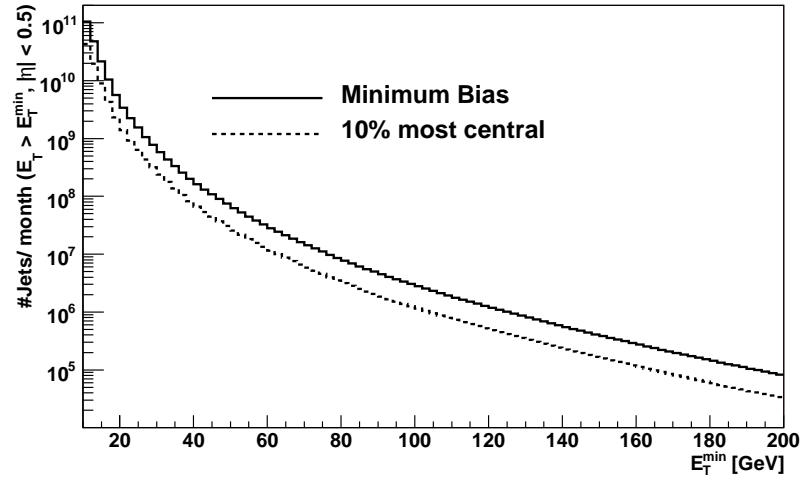

Fig. 1. Predicted annual jet yield vs. energy with $|\eta|<0.5$ in $\mathrm{PbPb}$ collisions at $\sqrt{s_{\mathrm{NN}}}=5.5 \mathrm{TeV}$.

pp collisions are defined as an excess of transverse energy over the background of the underlying event within a typical cone radius $R=1$ in the $\eta-\phi$ plane. $R=\sqrt{\Delta \eta^{2}+\Delta \phi^{2}}$ defines the geometrical size of the jet. In heavy-ion collisions at the LHC, the total energy from the underlying event in a cone of $R=1$ is expected to be of the order of $2 \mathrm{TeV}$, assuming $d N / d \eta=5000$, which is one order of magnitude higher than the highest jet energy we want to measure. Also, this energy fluctuates by an energy which is of the order of the jet energy. These two main limitations make jet identification in large cones in heavy ion collisions impossible.

However, if we take into account that about $80 \%$ of the jet energy is inside a cone of $R=0.3$ and that the background energy scales proportional to $R^{2}$ and its fluctuations proportional to $R$, we can reduce the contributions from the underlying event and its fluctuations by reducing the cone size to 0.3 or 0.4 . Another way of removing contribution of particles from the background is by applying a transverse momentum cut to the particles inside the cone, i.e. by removing low-momentum particles from the cone. These cuts may also reduced the signal but they do it to a much lesser extent.

Figure 2 compares the background energy from charged particles within a cone of size $R$ for different $p_{\mathrm{T}}$ thresholds to the energy from jets of different energies. For $R=1$, the background energy exceeds the jet energy even for the highest jet energy considered in the figure. It is also clear from this figure that the background energy can be reduced by reducing the cone size, by applying a $p_{\mathrm{T}}$ cut to the particles inside the cone, or by doing both.

However this method has its intrinsic performance limitations. Figure 3 shows the spectra of reconstructed cone energies for monoenergetic jets of $E_{\mathrm{T}}=100 \mathrm{GeV}$ generated by the Pythia event generator 14 and reconstructed using a cone size of $R=0.4$. The limited cone size leads to a low energy tail even for the best case scenario in which jets are reconstructed using charged and neutral tracks as measured by the central barrel tracking system and the electromagnetic calorimeter. When jets are reconstructed using only charged particles, the reconstruction is dominated by the charged to neutral fluctuations which results 


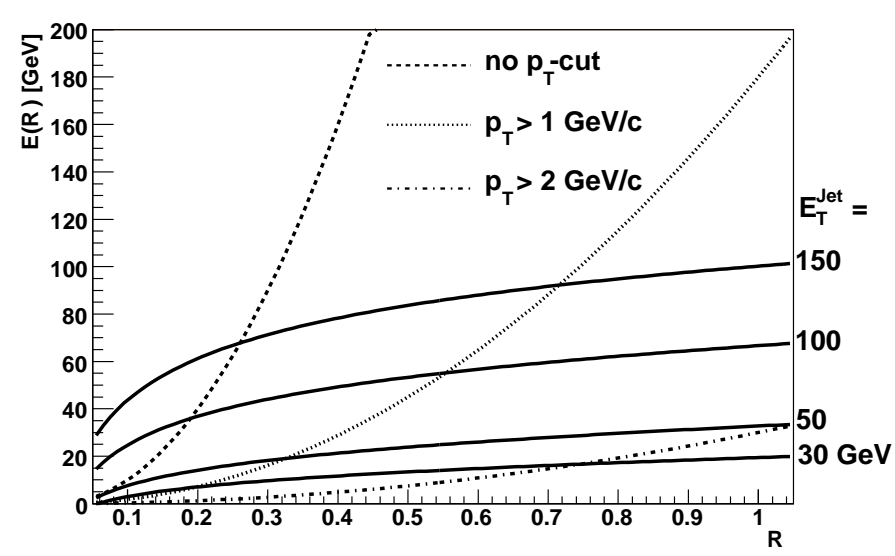

Fig. 2. Background energy from charged particles within a cone of size $R$ compared to the energy from jets of different energies for different transverse momentum thresholds.

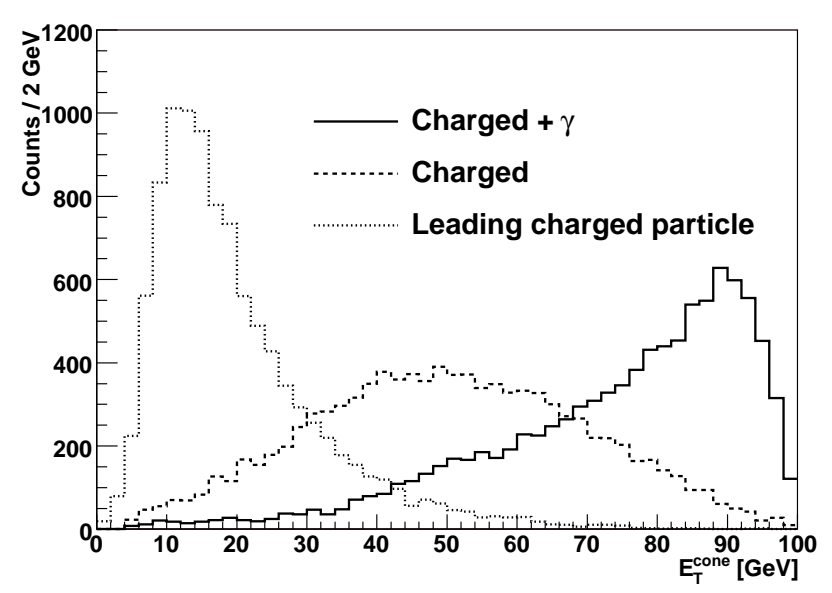

Fig. 3. Spectra of reconstructed cone energy for 10000 generated jets with $E=100 \mathrm{GeV}$ for different detector configurations. A cone of size $R=0.4$ was used.

in an almost Gaussian shape with a mean value at about half the input energy $\left(\Delta E_{\mathrm{T}} / E_{\mathrm{T}} \sim 50 \%\right)$.

\section{Jet Structure Observables}

ALICE has studied the reconstruction of jets by simulating jet events with the Pythia event generator and embedding them into an underlying $\mathrm{PbPb}$ event generated by the Hijing event generator [15]. The generated events were passed to the transport code GEANT3 [16] which simulates the trajectory of particles, their decays, and their interaction with the detector materials. Signal and underlying events were then merged and passed to the ALICE reconstruction algorithm for full reconstruction.

Two events samples were produced, one containing unquenched events and another one containing quenched events. In the latter case, energy loss was introduced in the Pythia simulations according to the toy model de-

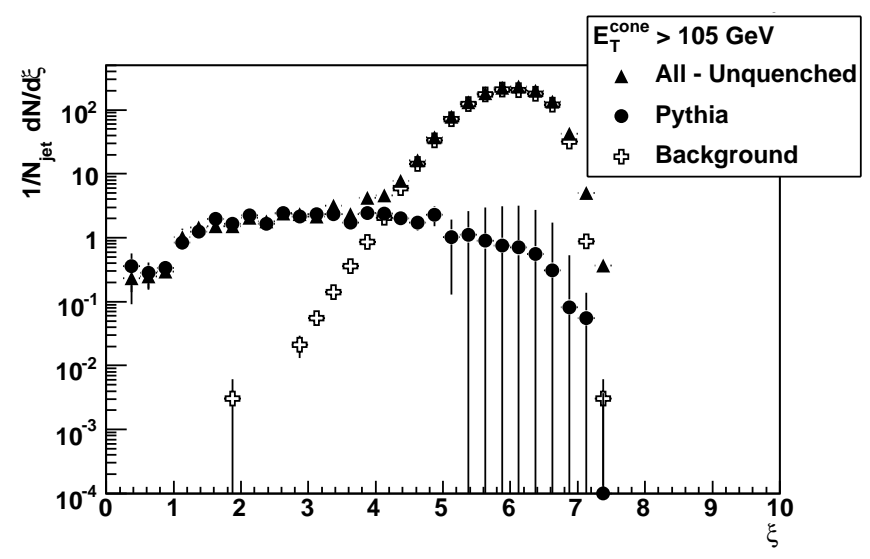

Fig. 4. Reconstructed hump-backed plateau for reconstructed energy $E_{\mathrm{T}}^{\text {cone }}>105 \mathrm{GeV}$. The spectra is compared to the corresponding result for background free events and an estimate of the background distribution.

scribed in [17. An average transport coefficient $\langle\hat{q}\rangle=$ $1.7 \mathrm{GeV}^{2} / \mathrm{fm}$ was used. Each event sample covers 13 bins $\left[p_{\mathrm{T}}^{\text {hard }}(i), p_{\mathrm{T}}^{\text {hard }}(i+1)\right]$, with $p_{\mathrm{T}}^{\text {hard }}(i+1) / p_{\mathrm{T}}^{\text {hard }}(i)=1.2$ from $20 \mathrm{GeV}$ to $180 \mathrm{GeV}$. Here $p_{\mathrm{T}}^{\text {hard }}$ is the transport momentum of the partons in the rest frame of the hard interaction.

We used our cone algorithm to identify and reconstruct jets in these simulated central $\mathrm{PbPb}$ events $(d N / d \eta=$ 5000). The cone radius was $R=0.4$ and the transverse momentum threshold $p_{\mathrm{T}}^{\mathrm{min}}=2 \mathrm{GeV}$. After jets are reconstructed, one can study the effects of the dense medium on the propagation of high energy partons or their decay products by studying the modification on the longitudinal and transverse momentum of the jet, i.e. through the modifications to the jet structure observables. In this section we describe different observables and present their analysis from the generated unquenched events mentioned above. A total of 3000 events per bin of $p_{T}^{\text {hard }}$ were used. For this jet-structure analysis, all particles inside the cone identified to be a jet were used, even those with $p_{\mathrm{T}}<2 \mathrm{GeV}$.

\subsection{Fragmentation Function}

A convenient way of representing the fragmentation function is through the distribution of $\xi=\ln \left(E_{\mathrm{T}}^{\mathrm{jet}} / p_{\mathrm{T}}^{\text {part }}\right)$. The characteristic shape of this distribution is known as the hump-backed plateau [18. Medium induced energy loss distorts the shape of this plateau in a characteristic way. This distortion is caused by the decrease on the number of particles with high $z$ and by the increase on the number of particles with low $z$.

Figure 4/shows the reconstructed hump-backed plateau for reconstructed energy $E_{\mathrm{T}}^{\text {cone }}>105 \mathrm{GeV}$ compared to the corresponding result for background free events. Also shown in the figure is an estimate of the background distribution. The region $\xi<4$ corresponds to $p_{\mathrm{T}} \gtrsim 1.8 \mathrm{GeV} / c$ and therefore leading particle remnants will appear in this region where the signal to background ratio $S / B$ is always 


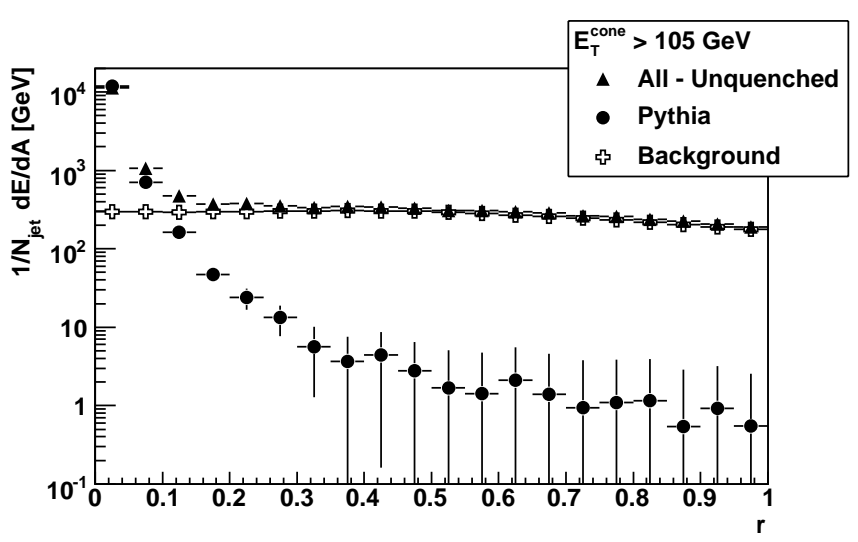

Fig. 5. Reconstructed distribution of charged energy averaged over rings $[R, R+\Delta R]$ around the jet axis normalised to the area $A$ of the ring for reconstructed energy $E_{\mathrm{T}}^{\text {cone }}>105 \mathrm{GeV}$. The spectra is compared to the corresponding result from background free events and an estimation of the background distribution.

larger than 0.1 . Therefore we will be able to measure modifications in the number of particles with high $z$. Particles from medium-induced gluon radiation are expected to appear predominantly in the region $4<\xi<6$. In this region $S / B$ is of the order of $10^{-2}$ making the study of particles with low $z$ more difficult.

\subsection{Jet Shape}

Jet shapes are described by the distribution of the average fraction of energy in a subcone of radius $r$,

$$
\Psi(r)=\frac{1}{N_{\text {jet }}} \sum \frac{p_{\mathrm{T}}(0, r)}{p_{\mathrm{T}}(0, R)},
$$

where $R$ is the jet size.

It is clear that lowering the momenta parallel to the jet axis and increasing the one perpendicular to it leads to an increase on the jet size. This increase is reflected in the jet shape. Calculations performed for a modest transport coefficient $\left(\hat{q} \sim 2 \mathrm{GeV}^{2} / \mathrm{fm}\right)$ show that the energy inside a cone of $R=0.4$ is reduced by about $5 \%$ for a jet of 50 $\mathrm{GeV}$ and by $3 \%$ for a jet of $100 \mathrm{GeV} 10$.

Figure 5 shows the distribution $\left(1 / N_{\mathrm{Jet}}\right) d E / d R$ averaged over rings $[R, R+\Delta R]$ around the jet axis normalised to the area $A$ of the ring for reconstructed energy $E_{\mathrm{T}}^{\text {cone }}>$ $105 \mathrm{GeV}$ compared to the corresponding result from background free events. An estimation of the background distribution is also shown in the figure. A clear excess of energy over the background close to the jet axis is seen. Observing energy of the order of few $\mathrm{GeV}$ radiated outside a cone of $R=0.4$ will be a challenge since the signal to background ratio decrease rapidly to $10^{-2}$ for larger radii.

\subsection{Momentum transverse to the jet axis}

The distribution of the momentum perpendicular to the jet axis or $j_{\mathrm{T}}$-distribution for jets can be measured in any

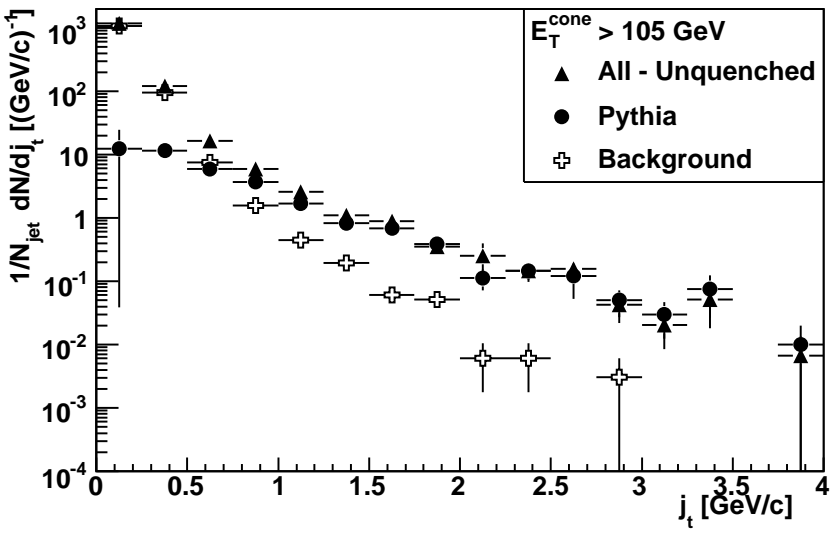

Fig. 6. Reconstructed $j_{\mathrm{T}}$-distribution for reconstructed energy $E_{\mathrm{T}}^{\text {cone }}>105 \mathrm{GeV}$. Only particles within $R<0.4$ entered the distribution. It is compared to the corresponding result for background free events and an estimation of the background distribution.

sub-cone region around the jet axis. Uncorrelated particles from the underlying event need a large angle with respect to the jet axis on order to have a large momentum perpendicular to the jet axis. Therefore, measuring the $j_{\mathrm{T}}$-distribution within a small radius preserves most of the signal and reduces significantly the background. What one expects to measure is a broadening of this distribution as well as an increase on its mean value due to the extra particles produced by the radiated gluons at low $j_{\mathrm{T}}[10$.

Figure 6] shows the reconstructed $j_{\mathrm{T}}$-distribution for reconstructed energy $E_{\mathrm{T}}^{\text {cone }}>105 \mathrm{GeV}$ compared to the corresponding result for background free events. An estimation of the background distribution is also shown in the figure. The expected high- $j_{\mathrm{T}}$-tail is observed.

\section{Leading Particle Correlations}

ALICE will also study the correlation between leading particles. This is an important analysis for two main reasons. While the event-by-event reconstruction of jets will be feasible for jet energies $E_{\mathrm{T}}>40 \mathrm{GeV}$, leading hadron correlations will be in principle possible to very low transverse momenta. Also, as shown in section 2 this kind of analysis has been performed at lower energies, therefore we will be able to directly compare our results with the results from RHIC that indicate a strong suppression of the leading particles of the recoiling jet.

\section{Conclusion}

With the copious production of jets in $\mathrm{PbPb}$ collisions at the LHC, ALICE will be able to identify jets using a reduced cone of $R=0.3-0.5$ in order to reduce contributions from the background. The properties of the created medium will then be studied through the jet structure observables. We have shown that jet-structure observables 
can actually be extracted from the sample of reconstructed jets and that the expected characteristics are seen: particles carrying a large fraction of the jet energy (low $\xi$ ), the high- $j_{\mathrm{T}}$-tail and the excess of energy close to the jet axis are clearly visible in our analysis and agree with the corresponding characteristics obtained from background-free events.

\section{References}

1. X.-N. Wang and M. Gyulassy, Nucl. Phys. B420 (1994) 583.

2. X.-N. Wang, M. Gyulassy and M. Plümer, Phys. Rev. D51, (1995) 3436.

3. J. Adams et al. [STAR Collaboration], Phys. Rev.Lett. 91 (2003) 072304.

4. B. B. Back et al. [PHOBOS Collaboration], Phys. Rev. Lett. 91 (2003) 072302.

5. S. S. Adler et al. [PHENIX Collaboration], Phys. Rev. Lett. 91 (2003) 072303.

6. I. Arsene et al. [BRAHMS Collaboration], Phys. Rev. Lett. 91 (2003) 072305.

7. R. Baier, Yu L. Dokshitzer, A. H. Mueller, S. Peigné and D. Schiff, Nucl. Phys. B484 (1997) 265.

8. M. Gyulassy, I. Vitev, X.-N. Wang and B.-W. Zhang, nucl-th/0302077 (2003).

9. K. J. Eskola, H. Honkanen, C. A. Salgado and U. A. Wiedemann, Nucl. Phys. A747 (2005) 51.

10. C. Salgado and U. A. Wiedemann, Phys. Rev. Lett. 93 (2004) 042301.

11. ALICE Collaboration, Technical Proposal, CERN/LHCC/95-71 (1995).

12. ALICE Collaboration, ALICE Physics Performance Report - Vol. 2, CERN/LHCC/2005-030 (2005), submitted to J. Phys. G.

13. T. M. Cormier, Eur. Phys. J. C34 (2004) S333.

14. T. Sjostrand, Phys. Lett. B157 (1985) 321.

15. X.-N. Wang and M. Gyulassy, Phys. Rev. D44 (1991) 3501.

16. R. Brun et al., GEANT3 User Guide, CERN Data Handling Division DD/EE/84-1 (1985).

17. J. Contreras, M. López Noriega and A. Morsch, ALICE Internal Note ALICE-INT-2005-035, (2005).

18. N. Borghini and U. A. Wiedemann, CERN-PH-TH-2005100, hep-ph/0506218 (2005). 